Evolution of ultrasonic impulses in chains of spheres using resonant excitation

This content has been downloaded from IOPscience. Please scroll down to see the full text. 2015 EPL 10954002

(http://iopscience.iop.org/0295-5075/109/5/54002)

View the table of contents for this issue, or go to the journal homepage for more

Download details:

IP Address: 109.146.78.115

This content was downloaded on 16/06/2016 at 15:56

Please note that terms and conditions apply. 


\title{
Evolution of ultrasonic impulses in chains of spheres using resonant excitation
}

\author{
D. A. Hutchins ${ }^{1}$, J. Yang ${ }^{1}$, O. Akanji ${ }^{1}$, P. J. Thomas ${ }^{1}$, L. A. J. Davies ${ }^{1}$, S. Freear ${ }^{2}$, S. Harput ${ }^{2}$, \\ N. SAFFARI ${ }^{3}$ and P. GELAT ${ }^{3}$ \\ 1 School of Engineering, University of Warwick - Coventry CV4 7AL, UK \\ 2 School of Electronic and Electrical Engineering, University of Leeds - Leeds LS2 9JT, UK \\ 3 Department of Mechanical Engineering, University College London - Torrington Place, London WC1E $7 J E$, UK
}

received 14 January 2015; accepted in final form 20 February 2015

published online 10 March 2015

PACS 43.35. $+\mathrm{d}-$ Acoustics: Ultrasonics, quantum acoustics, and physical effects of sound

PACS 46.40.-f - Vibrations and mechanical waves

PACS 45.50.-j-Dynamics and kinematics of a particle and a system of particles

\begin{abstract}
It is demonstrated that broad-bandwidth ultrasonic signals containing frequency components in excess of $200 \mathrm{kHz}$ can be created in spherical chains using harmonic excitation at $73 \mathrm{kHz}$. Multiple reflections created a periodic waveform containing both harmonics and subharmonics of the original forcing frequency, due to non-linear Hertzian contact. These discrete frequencies represented some of the many allowed non-linear normal modes of vibration of the whole chain. Excitation at a single fixed frequency could thus be used to produce wide-bandwidth impulses for different lengths of spherical chains. Experimental results were in good agreement with theoretical predictions.
\end{abstract}

open Caccess Copyright (c) EPLA, 2015

Published by the EPLA under the terms of the Creative Commons Attribution 3.0 License (CC BY). Further distribution of this work must maintain attribution to the author(s) and the published article's title, journal citation, and DOI.

Introduction. - The study of acoustic wave propagation in chain-like materials has shown that some distinctive properties arise [1,2]. Hertzian contact between spheres or other particles can lead to non-linear behaviour. In addition, the presence of dispersion in such systems means that solitary waves can be created [1], provided that the balance between dispersion and non-linearity is correct [3]. Non-linearity leads to interesting effects in terms of the normal modes of vibration (or resonances) of such chains $[4,5]$. Jayaprakash et al. [5] predicted the presence of non-linear normal modes (NNMs) of vibration within resonant chains. Two types of frequency bands were identified: propagation bands ( $\mathrm{PB}$ - allowed) and attenuation bands (AB - disallowed). This was a function of input energy level and the mode of vibration present (either in phase or out of phase).

The wave propagation characteristics in a particular chain depend upon many factors, such as the amplitude and frequency content of the input signal (in terms of an applied dynamic force $F_{m}$ ), the diameter of the spheres, and the relative values of $F_{m}$ and any pre-compression force $\left(F_{0}\right)$. Spadoni and Daraio [6] demonstrated that interesting effects occur in chains of stainless-steel spheres held within columns and put under variable pre-compression forces. The columns were then impacted with a transient force at one end. The properties of the signal output at the far end of the chain could be adjusted by changing the parameters of excitation and static pre-compression of the stack of spheres. When $F_{m} \gg F_{0}$, the value of $F_{0}$ could be used to adjust the propagation velocity, leading to the possibility of focussing and the concept of a sound bullet. A later paper extended the process, using more convenient piezoelectric actuation [7].

In the present work, large amplitudes available from resonant ultrasonic sources have been used as the source of harmonic inputs into chains of spheres to give high values of $F_{m}$. Travelling ultrasonic wave impulses are then created, due to both non-linearity and dispersion between adjacent spheres, and reflection within the chain, the latter acting as a system with non-linear resonant modes. The resulting impulses have both wide bandwidth and high amplitude, at frequencies in excess of $200 \mathrm{kHz}$. 
Voltage input

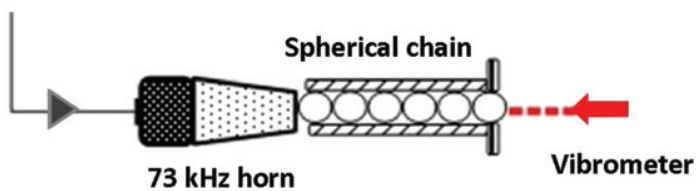

Fig. 1: (Colour on-line) Schematic diagram of the apparatus.

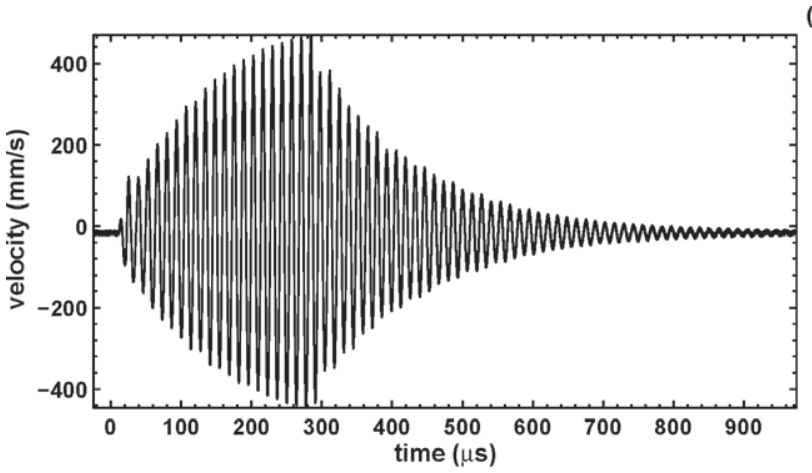

(b)

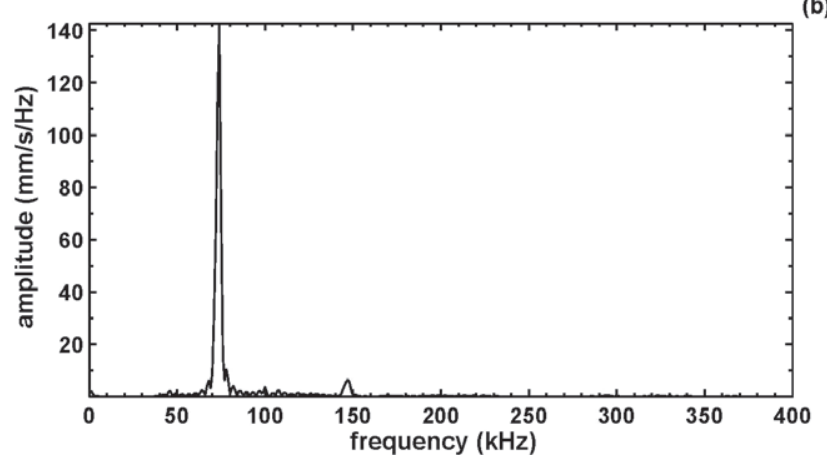

Fig. 2: (a) Particle velocity waveform and (b) corresponding spectrum (bottom) for vibrations at the horn tip at the maximum excitation voltage level.

Apparatus and experiment. - A schematic diagram of the experimental arrangement is shown in fig. 1. An ultrasonic transducer/horn arrangement was constructed, to produce high ultrasonic amplitudes, and hence large driving forces $\left(F_{m}\right)$ for input into the chain of spheres. The horn was driven into vibration using a tunable tone-burst voltage waveform, typically using a 20-cycle tone-burst drive signal at $73 \mathrm{kHz}$. This produced a maximum peak velocity at the horn tip of $0.45 \mathrm{~ms}^{-1}$, corresponding to a peak-to-peak displacement of $2 \mu \mathrm{m}$. This tip was then positioned so as to just touch one end of a set of spherical chrome steel spheres of $1 \mathrm{~mm}$ diameter, held horizontally within a cylindrical holder, as shown in fig. 1. Chain lengths of 6 and 10 spheres were tested initially. Ultrasonic coupling gel was used between the horn tip and the first sphere to minimise pre-compression force $F_{0}$. The holder had a plate at the far end, containing a $0.6 \mathrm{~mm}$ diameter aperture, through which the outermost sphere could protrude. The ultrasonic waveform at both the horn tip and at the output sphere at the far end of the chain was recorded in terms of particle velocity using a
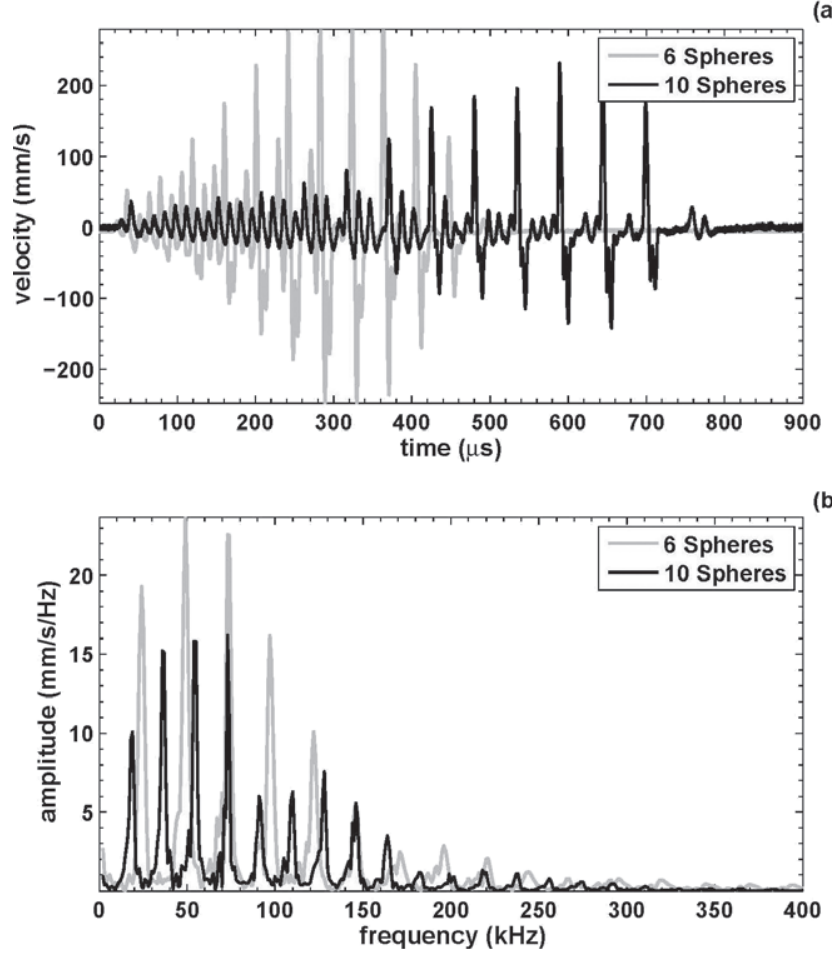

Fig. 3: (a) Waveform and (b) spectrum of signals generated experimentally within chains of both 6 and 10 spheres of radius $R=0.5 \mathrm{~mm}$ using the resonant horn at $73 \mathrm{kHz}$.

Polytec OFV 5000 vibrometer. The signals were recorded as a function of the input waveform $\left(F_{m}\right)$. Measurements of the resonant horn tip (fig. 2) showed a signal with frequencies predominantly at $73 \mathrm{kHz}$, with small amounts of harmonic content.

Experimental results. - It was observed experimentally that, as the amplitude of the input $F_{m}$ increased, both harmonics and sub-harmonics were observed to become more prominent. Example waveforms for chains of both 6 and 10 spheres are shown in fig. 3(a), in terms of the particle velocity amplitude $\left(v_{m}\right)$ waveform, with their corresponding spectra (obtained via an FFT) shown in fig. 3(b). This used an input signal at $73 \mathrm{kHz}$, of the form shown earlier in fig. 2.

Consider first the time waveform for the 6 -sphere chain (fig. 3(a)). Although some of the original input frequency of $73 \mathrm{kHz}$ dominated at the start of the arrival, a set of impulsive signals then built up, with a periodicity of $41 \mu \mathrm{s}$. These represent multiple reflections within the chain, with an acoustic group propagation velocity of $c_{g}=293 \mathrm{~ms}^{-1}$. These are the expected solitary wave impulses, which gain energy and build up amplitude from the original forcing frequency of the input, but then decay due to damping once the forcing function stops. The periodicity within the waveform is associated with the lowest sub-harmonic at $24.3 \mathrm{kHz}$ in the spectrum of fig. $3(\mathrm{~b})$, which is also the fundamental resonance of the chain. It is also at $1 / 3$ of the original forcing frequency of $73 \mathrm{kHz}$. Prominent harmon- 
ics also exist, that extend beyond $200 \mathrm{kHz}$, and these are also separated in frequency by a value of $1 / 3$ of the input frequency. The frequency peaks thus correspond to some of the many allowed non-linear normal modes (NNMs) of the system, and this is consistent with the propagation band (PB) concept described in Jayaprakash et al. [5]. It is this use of the resonant NNMs of the chain to allow impulses to be generated that is the distinguishing feature of this present approach.

The experimental waveform for the longer 10-sphere chain, also shown in fig. 3(a), demonstrates that in this case the impulses gradually built up over a longer timescale, before again decaying away once the excitation was removed. Note that the input frequency of $73 \mathrm{kHz}$ was a prominent peak for both chain lengths, as would be expected. However, the frequency spectrum of the longer chain contained three sub-harmonics, instead of the two seen for the 6-sphere case. The fundamental resonance was now at a lower frequency of $18.25 \mathrm{kHz}$ (i.e. $1 / 4$ that of the input). The corresponding group velocity of the impulses was $364 \mathrm{~ms}^{-1}$, an increase compared to that measured in the 6 -sphere chain. The harmonic content also increased in the longer chain. The result can be interpreted as the system now selecting a different fundamental NNM of resonance within the $\mathrm{PB}$ regime than was present for a chain with 6 spheres. It is interesting to note that for both the 6-sphere and 10-sphere chains, the lowest resonant spectral peak, in each case, is a sub-harmonic of the original forcing frequency (at $1 / 3$ and $1 / 4$ of this value, respectively). Hence, in both cases, energy can be efficiently transferred from the input frequency into the various NNM resonances within each chain. This, in turn, allows the creation of wide-bandwidth impulses.

Experiments at other chain lengths - as well as concurrent simulations of the type to be outlined belowhave shown that the behaviour described above for 6 and 10 spheres is often not obtained. For example, systems with 7,8 and 9 spheres showed only the creation of harmonics due to the expected non-linear Hertzian behaviour between the successive chain elements. It would thus appear that a certain combination of factors including the input signal and the physical properties of the chain of spheres is necessary to obtain the resonant behaviour and, hence, the creation of reflecting impulses.

Comparison to theory. - The above results can be compared to theory using established modelling approaches for wave propagation in a single chain of spheres $[1,8,9]$. These spheres are assumed to be touching, and a static pre-compression can be applied if required. The system can be considered to be displacement driven [8], and this displacement $u_{0}$ can be calculated by the velocity that would be measured experimentally at the horn tip, which is assumed to have planar surface contact with the spheres. At the far end of the chain, the motion of the last sphere can be predicted, assuming that it is in contact with a fixed planar wall (the holder used to con- tain the spheres). Thus, both contact between the spheres, and the interactions between spheres and materials at either end of the chain, can be modelled. The starting point of the theoretical formulation is the classic Hertzian 3/2 power law (cf. ref. [1]) for the elastic force $F$ between two spheres under compression. The displacements of the centre of the spheres in a chain of length $N$ are denoted as $u_{i}$ with $(i=1 \ldots N)$. Assuming that all spheres have the same radius $R$ and the same material properties, the dynamic equations of motion can be determined. The model can also consider the effect of the flat surfaces at each end of the chain (the horn tip at the input and the container wall at the output). A static applied force $F_{0}$ can be modelled by deriving the initial static displacements between the horn and the centre of the first sphere $\left(\delta_{0 l}\right)$, between successive sphere centres $\left(\delta_{0}\right)$, and between the last sphere and the holder wall $\left(\delta_{0 r}\right)$. The equation for the input sphere (nearest the horn) is

$$
\begin{aligned}
m \frac{\mathrm{d}^{2} u_{1}}{\mathrm{~d} t^{2}}= & \frac{2 \sqrt{R}}{3}\left[2 \theta_{l}\left(\delta_{0 l}+u_{0}-u_{1}\right)^{\frac{3}{2}}-\frac{\theta_{m}}{\sqrt{2}}\left(\delta_{0}+u_{1}-u_{2}\right)^{\frac{3}{2}}\right] \\
& +\lambda\left(\frac{\mathrm{d} u_{0}}{\mathrm{~d} t}-\frac{\mathrm{d} u_{1}}{\mathrm{~d} t}\right) H\left(\delta_{0 l}+u_{0}-u_{1}\right) \\
& -\lambda\left(\frac{\mathrm{d} u_{1}}{\mathrm{~d} t}-\frac{\mathrm{d} u_{2}}{\mathrm{~d} t}\right) H\left(\delta_{0}+u_{1}-u_{2}\right)
\end{aligned}
$$

For subsequent spheres, up to and including the penultimate one, the equation is

$$
\begin{aligned}
m \frac{\mathrm{d}^{2} u_{i}}{\mathrm{~d} t^{2}}= & \frac{\sqrt{2 R}}{3} \theta_{m}\left[\left(\delta_{0}+u_{i-1}-u_{i}\right)^{\frac{3}{2}}-\left(\delta_{0}+u_{i}-u_{i+1}\right)^{\frac{3}{2}}\right] \\
& +\lambda\left(\frac{\mathrm{d} u_{i-1}}{\mathrm{~d} t}-\frac{\mathrm{d} u_{i}}{\mathrm{~d} t}\right) H\left(\delta_{0}+u_{i-1}-u_{i}\right) \\
& -\lambda\left(\frac{\mathrm{d} u_{i}}{\mathrm{~d} t}-\frac{\mathrm{d} u_{i+1}}{\mathrm{~d} t}\right) H\left(\delta_{0}+u_{i}-u_{i+1}\right)
\end{aligned}
$$

Finally, for the last sphere next to the container wall the equation is

$$
\begin{aligned}
m \frac{\mathrm{d}^{2} u_{N}}{\mathrm{~d} t^{2}}= & \frac{2 \sqrt{R}}{3}\left[\frac{\theta_{m}}{\sqrt{2}}\left(\delta_{0}+u_{N-1}-u_{N}\right)^{\frac{3}{2}}-2 \theta_{r}\left(\delta_{0 r}+u_{N}\right)^{\frac{3}{2}}\right] \\
& +\lambda\left(\frac{\mathrm{d} u_{N-1}}{\mathrm{~d} t}-\frac{\mathrm{d} u_{N}}{\mathrm{~d} t}\right) H\left(\delta_{0}+u_{N-1}-u_{N}\right) \\
& -\lambda \frac{\mathrm{d} u_{N}}{\mathrm{~d} t} H\left(\delta_{0 r}+u_{N}\right),
\end{aligned}
$$

where $\theta_{m}, \theta_{r}$ and $\theta_{l}$ are given by

$$
\begin{aligned}
\theta_{m} & =\frac{E_{s}}{1-\nu_{s}^{2}}, \\
\frac{1}{\theta_{r}} & =\frac{1-\nu_{r}^{2}}{E_{r}}+\frac{1-\nu_{s}^{2}}{E_{s}}, \\
\frac{1}{\theta_{l}} & =\frac{1-\nu_{l}^{2}}{E_{l}}+\frac{1-\nu_{s}^{2}}{E_{s}},
\end{aligned}
$$

where $E_{s}$ and $\nu_{s}$ are Young's modulus and Poisson's ratio of the spheres, $E_{r}$ and $\nu_{r}$ those of the end wall, and $E_{l}$ and 
$\nu_{l}$ those of the horn, respectively. Note that only positive arguments for the 3/2-power-law terms in eq. (1) have to be considered, as these are associated with the spheres being in contact. The terms are set to zero for negative values, i.e. tensionless behaviour, when the spheres lose contact.

The theory can now be used to evaluate the expected waveform for a given dynamic input force $F_{m}$. It should be noted that the modelling of such non-linear systems is inherently highly sensitive to the input and boundary conditions, so that exact agreement between theory and experiment can be difficult. However, we show good agreement to the experimental observations. In order to model the experiments more accurately we follow Lydon et al. [8] and introduce a linear viscous damping coefficient $\lambda$ between successive spheres in the dynamic equations. The damping force is only relevant when the spheres are in contact, and was needed to eliminate high-frequency chaotic instability from the dynamics. Note that damping was evident in the experimental waveforms, making this a reasonable approach.

The particle velocity waveforms, calculated for the two spherical chains with a small static applied force $(<0.1 \mathrm{~N})$ and a linear viscous damping coefficient $\lambda=0.27 \mathrm{Nsm}^{-1}$, are shown in fig. 4(a). The input signal $\left(F_{m}\right)$ used in the model was that measured experimentally for the horn tip in each case, an example of which was shown earlier in fig. 2. In both cases, the theoretical prediction is for a set of impulses which build up with time. In agreement with experiment, the impulses take more time to establish for the longer 10-sphere chain, with an increased periodicity. The frequency spectra (fig. 4(b)) both contain a peak at the input frequency of $73 \mathrm{kHz}$, plus prominent sub-harmonics and higher-order harmonics. Again in agreement with experiment, the lowest (fundamental) frequency peak in each case is predicted to be at $1 / 3$ and $1 / 4$ of the input frequency for the 6 -sphere and 10 sphere chains, respectively, with a different group velocity of propagation along the chain. The relative amplitude of each harmonic is not quite the same as in the experiment, but modelling has predicted the main features of the process.

Discussion. - The results shown above are compatible with the known behaviour of acoustic propagation along spherical chains [1], namely that the interaction between spheres is highly non-linear, due to Hertzian contact, and dispersive. In this particular example, the spheres were chosen to have a smaller diameter than in many other studies [6-9] so that the expected behaviour could be observed conveniently in the ultrasonic range.

At the high input signal levels $\left(F_{m}\right)$ used, the input energy was sufficient to generate both harmonics and subharmonics. This situation exhibits the expected pass band (PB) behaviour described by Jayaprakash et al. [5]. The results of fig. 3 demonstrated that impulses could be created by multiple reflection within two different
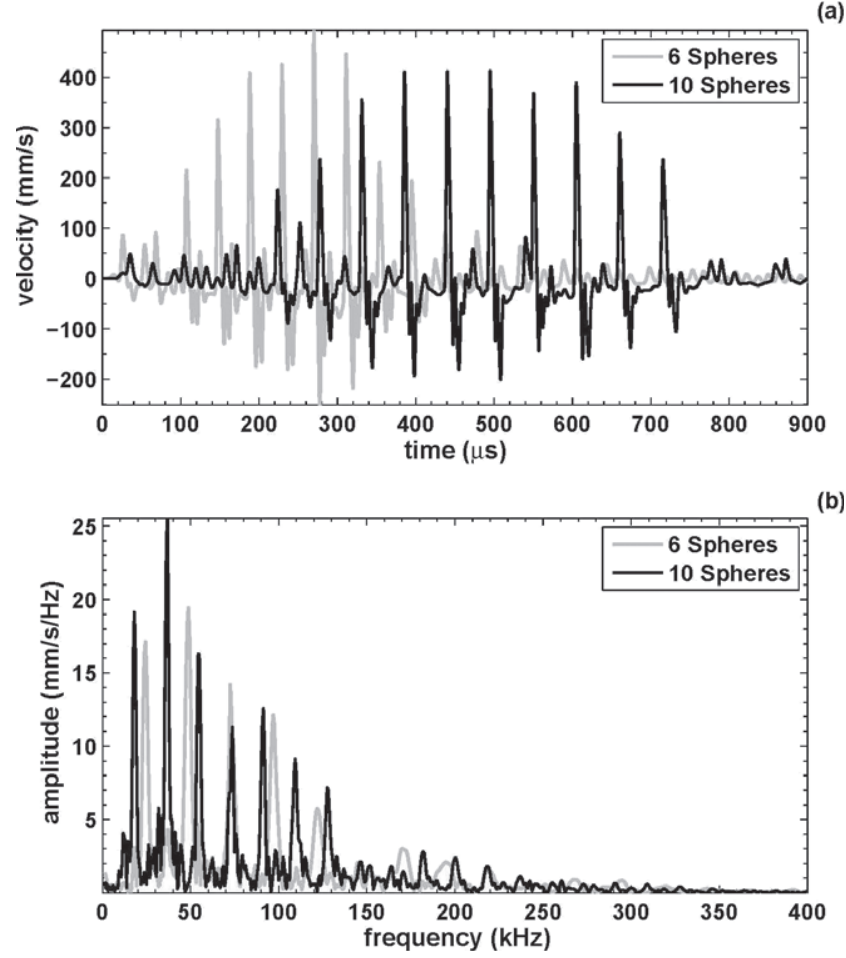

Fig. 4: Waveforms (top) and spectra (bottom) of waveforms predicted theoretically for the same conditions as those used experimentally in fig. 3 .

lengths of chain. Each impulse had a spectrum that was much broader than that of the original forcing signal. An important difference to previous studies is that the input was from a high-amplitude, highly-resonant excitation system, chosen to be of narrow bandwidth at $73 \mathrm{kHz}$; this maximised input energy. The observed build-up of solitary wave impulses was also predicted by the numerical simulations based on the discrete dynamic equations of spheres in Hertzian contact with one another. The fact that particular NNMs appear for sets of 6 and 10 spheres, but not for other systems with 7,8 and 9 spheres, for example, is one of the most interesting conclusions of this work. Given that in similar systems with steady harmonic excitation this type of resonant motion should also exist, it is likely that this finding is closely related to the specific time duration of the applied load. In fact, in other experiments we have observed that, for applied excitations of longer or shorter duration, the results change for a particular chain length. This is a topic for further investigation by the authors.

An interesting point in the present experiments is that impulses were generated only when particular forcing conditions, in terms of amplitude and time duration, were present for a particular forcing frequency $F_{m}$, at which point discrete frequency peaks also appeared. This, we believe, is an important factor in that it allows the natural build-up of energy into the solitary-wave impulses, by feeding energy into a set of natural frequencies of the system of spheres which are harmonics and sub-harmonics of 
$F_{m}$. This interesting observation is also consistent with the NNM theory outlined in refs. [4,5], in that when $F_{m} \gg F_{0}$, there are many allowed natural modes of the system, including both harmonics and sub-harmonics of $F_{m}$. The fact that a different group propagation velocity was observed for impulses within the two lengths of chain is also consistent with the NNM theory. The system could select a mode, from the many that were available; this resulted in a frequency separation between spectral peaks that was exactly either $1 / 4$ or $1 / 3$ that of the forcing frequency depending on the length of the chain. It is evident also from the experiments that the longer chain resulted in more closely-spaced harmonics which extended to higher frequencies. This would be expected due to the extended non-linearity in a longer chain.

Conclusion. - It has been shown experimentally that solitary wave impulses can be generated using highamplitude resonant excitation. The results are consistent with modelling studies, which show that the generation of solitary wave impulses depends on the exact nature of both the chain and the excitation waveform. When conditions are such that $F_{m} \gg F_{0}$, solitary wave impulses can be produced using sinusoidal excitation at $73 \mathrm{kHz}$, as observed by both experiments and numerical calculations. Comparison of the behaviour in the two chain lengths suggests that such a system could adapt to exist within many different chain configurations, provided that the system is consistent with the PB region for the non-linear normal modes of the chain.

The fact that only certain chain lengths show the behaviour demonstrated in fig. 3 and fig. 4 is interesting. Experiments with much longer chains of 20 spheres were not successful due to the high damping/attenuation of the high-frequency signals. Further modelling studies are needed to define the exact parameters that determine a successful resonant system. However, the modelling of the present arrangement indeed confirms that 6- and 10-sphere chains give the best response for the type of sphere chains considered in our present study.

$$
* * *
$$

The authors gratefully acknowledge funding from the Engineering and Physical Sciences Research Council (UK) via grant No. EP/K030159/1. Helpful discussions with Prof. RoBert MCKAY, Director of the Centre for Complexity Science at the University of Warwick, are gratefully acknowledged.

\section{REFERENCES}

[1] Nesterenko V. F., J. Appl. Phys., 24 (1983) 733.

[2] Sen S., Hong J., Avalos E. and Doney R., Phys. Rep., 462 (2008) 21.

[3] Nettles S., Wave Physics Oscillations-Solitons-Chaos, 4th edition (Springer) 2008.

[4] Starosvetsky Y. and Vakakis A. F., Phys. Rev. E, 82 (2009) 026603.

[5] Jayaprakash K. R., Starosvetsky Y., Vakakis A. F., Peeters M. and Kerschen G., Nonlinear Dyn., 63 (2011) 359.

[6] Spadoni A. and Daraio C., Proc. Natl. Acad. Sci. U.S.A., 107 (2010) 7230.

[7] Donahue C. M., Anzel P. W. J., Bonamoni L., Keller T. A. and Daraio C., Appl. Phys. Lett., 104 (2014) 014103.

[8] Lydon J., Jayaprakash K. R., NGo D., Starosvetsky Y., Vakakis A. F. and Daraio C., Phys. Rev. E, 88 (2013) 012206.

[9] Coste C., Falcon E. and Fauve S., Phys. Rev. E, 56 (1997) 6104 